\title{
Momentum density and its Fourier transform: Relation to the first-order density matrix and some scaling properties
}

\author{
I. A. Howard, ${ }^{1}$ N. H. March, ${ }^{1,2}$ and V. E. Van Doren ${ }^{1}$ \\ ${ }^{1}$ Department of Physics, University of Antwerp (RUCA), Groenenborgerlaan 171, B-2020 Antwerp, Belgium \\ ${ }^{2}$ Oxford University, Oxford, England \\ (Received 26 April 2001; published 17 September 2001)
}

\begin{abstract}
Density-functional theory requires knowledge of the kinetic-energy density $t(\mathbf{r})$ in terms of the ground-state density $\rho(\mathbf{r})$. Of course, the direct route to total kinetic energy is from the momentum density $n(\mathbf{p})$, which in turn is directly related by Fourier transform to the first-order density matrix $\gamma\left(\mathbf{r}, \mathbf{r}^{\prime}\right)$. Here, an alternative route to calculate the total kinetic energy is explored, via the Fourier transform $\widetilde{n}(\mathbf{r})$ of the momentum density $n(\mathbf{p})$. It is shown that $\widetilde{n}(\mathbf{r})$ is related to the density matrix $\gamma$ through its contracted form $\int \gamma\left(\mathbf{r}^{\prime}-\mathbf{r}, \mathbf{r}^{\prime}\right) d \mathbf{r}^{\prime}=\widetilde{n}(\mathbf{r})$. As examples, bare Coulomb field and harmonic confinement for arbitrary numbers of closed shells are treated. Finally, a localized potential $V(\mathbf{r})$ embedded in an initially uniform electron gas is considered, but now to low order in a perturbation series in $V(\mathbf{r})$.
\end{abstract}

DOI: 10.1103/PhysRevA.64.042509

PACS number(s): 31.15.Ew

\section{INTRODUCTION}

Density-functional theory, having its origins in the Thomas-Fermi (TF) statistical theory [1,2], requires knowledge of the kinetic energy $t(\mathbf{r})$ in terms of the ground-state electron density $\rho(\mathbf{r})$. In the range of validity of the TF method [3], the kinetic-energy density $t_{\mathrm{TF}}(\mathbf{r})$ is given by

$$
t_{\mathrm{TF}}(\mathbf{r})=c_{k}\{\rho(\mathbf{r})\}^{5 / 3}, \quad c_{k}=\frac{3 h^{2}}{10 m_{e}}\left(\frac{3}{8 \pi}\right)^{2 / 3} .
$$

In early work [4,5], it was shown that this statistical theory allowed direct calculation of the momentum density $n(\mathbf{p})$. Then the total kinetic energy is readily obtained from the second moment $\left\langle p^{2}\right\rangle$ of $n(\mathbf{p})$. In turn, the moments of $n(\mathbf{p})$ can be directly related to integrals on $\rho(\mathbf{r})$ in the ThomasFermi limit.

One of us [6] showed, for the model of closed shells in a bare Coulomb field, using a result going back to Fock [7] for the momentum density $n_{m}(\mathbf{p})$ of the $m$ th closed shell, namely,

$$
n_{m}(\mathbf{p})=\frac{16 m^{2} p_{m}^{5}}{\pi^{2}\left(p^{2}+p_{m}^{2}\right)^{4}} ; \quad p_{m}=Z / m
$$

that one could connect, in this specific example, fully quantum-mechanical results with the semiclassical TF theory.

Of course, it is well known [8,9] that one route to the momentum density $n(\mathbf{p})$ is via the first-order density matrix $\gamma\left(\mathbf{r}, \mathbf{r}^{\prime}\right)$. Unfortunately, to date, there are only a very limited number of examples, even for independent Fermions for which $\gamma$ is an idempotent matrix, in which closed analytic forms of $\gamma\left(\mathbf{r}, \mathbf{r}^{\prime}\right)$ can be written. One example, namely, harmonic confinement in one dimension, goes back to Husimi $[10,11]$. Another, the so-called Bardeen model $[12,13]$ of a metal surface in which otherwise free electrons are confined by an infinite (planar) barrier, has a $\gamma\left(\mathbf{r}, \mathbf{r}^{\prime}\right)$ which has been utilized recently [14] to obtain $t(\mathbf{r})$ and the corresponding exchange energy density in analytic form.
Here, we shall explore first an alternative route to the total kinetic-energy $T$, via the Fourier transform of the momentum density $n(\mathbf{p})$. This route is set out in Sec. II below. Section III considers the relatively simple example of isotropic harmonic confinement in three dimensions for an arbitrary number of closed shells, while Sec. IV deals with the corresponding problem for a bare Coulomb field, motivated by Fock's result (1.2). Following these two soluble bound-state problems, the case of a localized potential energy $V(\mathbf{r})$ inserted into an initially uniform electron gas is considered in Sec. V, but now only to lowest order in a perturbative development in $V(\mathbf{r})$. Section VI constitutes a summary, plus some proposals for further work while in Appendix A, the twoelectron Hookean atom, introduced by Kestner and Sinanoglu [15], is considered within the present framework, as probably the simplest example where electron-electron interaction can be handled analytically for special values of the Hooke's Law force constant.

\section{FOURIER TRANSFORM OF THE MOMENTUM DENSITY $n(p)$ AND ITS RELATION TO THE FIRST-ORDER DENSITY MATRIX $\gamma\left(\mathbf{r}, \mathbf{r}^{\prime}\right)$.}

The scattering factor $f(\mathbf{k})$, as is well known, is related to the ground-state density $\rho(\mathbf{r})$ by the Fourier transform relation

$$
f(\mathbf{k})=\int \rho(\mathbf{r}) \exp (i \mathbf{k} \cdot \mathbf{r}) d \mathbf{r} .
$$

For closed-shell atoms like $\mathrm{He}$ or $\mathrm{Ne}, f(\mathbf{k})$ is accessible experimentally via $\mathrm{x}$-ray diffraction. As to the momentum density $n(\mathbf{p})$, for similar closed-shell atoms it is also experimentally accessible by measuring the shape of the Compton line in x-ray scattering. A suitable reduced form, going back to Duncanson and Coulson [16], describes the line shape by a function $J(q)$, which is related to the probability $I(p) d p$ that an electron in such a closed-shell atom will have a momentum of magnitude lying between $p$ and $p+d p$. The precise relation is 


$$
J(q)=\frac{1}{2} \int_{q}^{\infty} \frac{I(p)}{p} d p .
$$

Motivated by Eqs. (2.1) and (2.2) with the addition

$$
I(p)=4 \pi p^{2} n(p),
$$

we now introduce the Fourier transform $\tilde{n}(\mathbf{r})$ of $n(\mathbf{p})$ through the definition

$$
\widetilde{n}(\mathbf{r})=\int n(\mathbf{p}) \exp \left(i \frac{\mathbf{p} \cdot \mathbf{r}}{\hbar}\right) d \mathbf{p} .
$$

Because of the importance of the kinetic-energy density $t(\mathbf{r})$ in current density-functional theory, we shall also consider the definition

$$
t(\mathbf{p})=\frac{p^{2}}{2 m_{e}} n(\mathbf{p})
$$

with $m$ the electron mass. Thus,

$$
n(\mathbf{p})=2 m_{e} \frac{t(\mathbf{p})}{p^{2}}
$$

and defining the Fourier transform of $t(\mathbf{p})$ by $\tilde{t}(\mathbf{r})$ through

$$
\tilde{t}(\mathbf{r})=\int t(\mathbf{p}) \exp \left(i \frac{\mathbf{p} \cdot \mathbf{r}}{\hbar}\right) d \mathbf{p}
$$

we have, by taking the Fourier transform of Eq. (2.6),

$$
\tilde{n}(\mathbf{r})=\frac{m_{e}}{2 \pi} \int \frac{\tilde{t}(\mathbf{r})}{\left|\mathbf{r}-\mathbf{r}^{\prime}\right|} d \mathbf{r}^{\prime}
$$

where the convolution property of the Fourier transform of a product has been utilized. Invoking at this stage the analogy between Eq. (2.8) and the solution of Poisson's equation of electrostatics for the potential in terms of the charge density, we readily obtain the relation (from this point, we work in atomic units throughout)

$$
\tilde{t}(\mathbf{r})=-\frac{1}{2} \nabla^{2} \tilde{n}(\mathbf{r})
$$

We shall exemplify this relation in Secs. III and IV for both harmonic confinement and the bare Coulomb potential. However, let us conclude this section by relating $\widetilde{n}(\mathbf{r})$ to the first-order density matrix $\gamma\left(\mathbf{r}, \mathbf{r}^{\prime}\right)$. We have the usual relation $[8,9]$

$$
n(\mathbf{p})=\int \gamma\left(\mathbf{r}, \mathbf{r}^{\prime}\right) \exp \left[i \mathbf{p} \cdot\left(\mathbf{r}-\mathbf{r}^{\prime}\right)\right] d \mathbf{r} d \mathbf{r}^{\prime}
$$

and, hence, it follows directly that

$$
\tilde{n}(\mathbf{r})=\int \gamma\left(\mathbf{r}^{\prime}-\mathbf{r}, \mathbf{r}^{\prime}\right) d \mathbf{r}^{\prime}
$$

This "contracted" form of the first-order density matrix therefore determines the Fourier transform $\widetilde{n}(\mathbf{r})$ of the momentum density. Evidently, putting $\mathbf{r}=0$ in Eq. (2.11), we find

$$
\tilde{n}(0)=\int \gamma\left(\mathbf{r}^{\prime}, \mathbf{r}^{\prime}\right) d \mathbf{r}^{\prime}=\int \rho\left(\mathbf{r}^{\prime}\right) d \mathbf{r}^{\prime}=N,
$$

where $N$ is the total number of fermions. Similarly, returning to Eq. (2.7), we see that

$$
\tilde{t}(0)=\int t(\mathbf{p}) d \mathbf{p}=T,
$$

where $T$ is the total kinetic energy.

The following three sections will be focused on giving explicit examples of these r-space quantities $\widetilde{n}(\mathbf{r})$ and $\tilde{t}(\mathbf{r})$, which are related by the basic Eq. (2.9). In Appendix B, we explicitly relate the Compton profile $J(q)$ to $\widetilde{n}(\mathbf{r})$.

\section{ISOTROPIC HARMONIC CONFINEMENT}

For the case of $N$ independent Fermions in an isotropic three-dimensional harmonic potential $V(r)=(1 / 2) m \omega^{2} r^{2}$, we have recently shown [17] that the total Fermion density $\rho(r)$ for $(M+1)$ closed shells can be expressed in terms of a relatively simple finite series. The symmetry of the Hamiltonia means that the wave functions for the three-dimensional harmonic oscillator have the same functional form in $\mathbf{p}$ and $\mathbf{r}$ space [18]; therefore, the total density $n(p)$ in $\mathbf{p}$ space for $(M+1)$ closed shells must have the same functional form as $\rho(r)$ in $\mathbf{r}$ space. So we may write (in atomic units)

$$
n(p)=\frac{N \omega^{-3 / 2}}{2 \pi} \exp \left(-p^{2} / \omega\right) \frac{\sum_{n=0}^{M} a(n)\left(p^{2} / \omega\right)^{n}}{\sum_{n=0}^{M} a(n) \Gamma(n+3 / 2)},
$$

where the $a(n)$ are related by

$$
\begin{aligned}
0= & a(n+2)\left[\frac{(n+2)(2 n+5)}{2}\right]+a(n+1)[2(M+1) \\
& -3(n+1)]+a(n)\left[\frac{2(n-M)}{(n+1)}\right], \quad a(M)=2^{M},
\end{aligned}
$$

and the total fermion number $N=(M+1)(M+2)(M$ $+3) / 6$. It is then possible to transform $n(p)$ to find $\widetilde{n}(\mathbf{r})$ as

$$
\tilde{n}(\mathbf{r})=N \frac{\sum_{n=0}^{M} a(n) \Gamma(n+3 / 2) \mathbf{M}\left(n+3 / 2,3 / 2,-\omega^{2} r / 4\right)}{\sum_{n=0}^{M} a(n) \Gamma(n+3 / 2)},
$$

where $\mathbf{M}(a, b, z)$ is Kummer's $\mathbf{M}$ function [19], and $\widetilde{t}(\mathbf{r})$ follows from Eqn. (2.9). Note that $\mathbf{M}(a, b, z) \rightarrow 1$ as $z \rightarrow 0$, so 
that $\widetilde{n}(0)=N$ as required. The Compton profile $J(q)$ can likewise be calculated analytically; with the momentum distribution $I(p)=4 \pi p^{2} n(p)$ determined by Eq. (3.1), we can evaluate $J(q)$ as

$$
\begin{aligned}
J(q)= & -\frac{N \omega^{-1 / 2}}{2} \exp \left(-q^{2} / \omega\right) \\
& \times \frac{\sum_{n=0}^{M} a(n)\left(q^{2} / \omega\right)^{n+1} \mathbf{M}\left(1, n+2, q^{2} / \omega\right) /(n+1)}{\sum_{n=0}^{M} a(n) \Gamma(n+3 / 2)} \\
& +\frac{N \omega^{-1 / 2}}{2} \frac{\sum_{n=0}^{M} a(n) \Gamma(n+1)}{\sum_{n=0}^{M} a(n) \Gamma(n+3 / 2)},
\end{aligned}
$$

where $\mathbf{M}(a, b, z)$ is as in Eq. (3.3). Equations (3.3) and (3.4) are clearly amenable to numerical study for small values of $M$. Also, we note that these two results for $\widetilde{n}(\mathbf{r})$ and $J(q)$ are related by the cosine transform exhibited in Eq. (B5).

\section{CLOSED SHELLS IN A BARE COULOMB FIELD: ESPECIALLY SCALING PROPERTIES}

While Fock's expression for $n_{m}$ (p) [Eq. (1.2)] can be summed over $m$ from one to $N$ for $N$ shells in terms of the polygamma functions, as we have shown elsewhere [20], we have subsequently obtained the limit $N \rightarrow \infty$ in terms of functions of $q=p / Z$. The result is

$$
\begin{aligned}
n_{\infty}(q, Z)= & \sum_{m=1}^{\infty} n_{m}(q, Z)=\frac{Z^{-3}}{3 \pi^{2}} q^{-5} \operatorname{Re}\left[-q^{2} \psi^{\prime \prime \prime}(i q)\right. \\
& \left.+3 q \psi^{\prime \prime}(i q)-3 \psi^{\prime}(i q)\right],
\end{aligned}
$$

(compare the r-space density given by Heilmann and Lieb [21]) where $\psi(z)=\partial[\ln \Gamma(z)] / \partial z$ is the Psi (Digamma) function, and primes denote differentiation with respect to $q$.

The property of this exact limiting result (4.1) that we wish to stress immediately is that $n_{\infty}(q, Z)$ takes the form

$$
n_{\infty}(p, Z)=Z^{-3} f_{\infty}(q) \text {. }
$$

Thus, by simple scaling with atomic number $Z$, a function of the variables $p$ and $Z$ is essentially reduced to the singlevariable function $f_{\infty}(q)$, which is immediately written down from Eq. (4.1). Motivated by this, we have returned to the expression for an arbitrary number $N$ of closed shells, and can show that it exhibits the scaling property

$$
n(N, Z, p)=\frac{16 Z^{-3}}{\pi^{2}} f(N, q),
$$

which, as for the limit $N \rightarrow \infty$ reported in Eq. (5) above, demonstrates that one thereby reduces a function of three variables, after scaling with $Z$, to what is essentially a func-
TABLE I. Moments of $f(q)$ for $N$ filled shells.

\begin{tabular}{rc}
\hline \hline$n$ & $\left\langle q^{n}\right\rangle$ \\
\hline-2 & $N\left(6 N^{4}+15 N^{3}+10 N^{2}-1\right) \pi / 192$ \\
-1 & {$[N(N+1)]^{2} / 24$} \\
0 & $N(N+1)(2 N+1) \pi / 192$ \\
1 & $N(N+1) / 24$ \\
2 & $N \pi / 32$ \\
3 & {$\left[\gamma+\psi_{1}(N+1)\right] / 6$} \\
4 & $5 \pi^{2}\left[1-6 \psi_{1}(N+1) / \pi^{2}\right] / 192$ \\
\hline \hline
\end{tabular}

tion of only two. We note that it follows from summing Eq. (1.2) over $N$ shells that in the limit $q \rightarrow 0, f(N, 0)=N^{2}(N$ $+1)^{2}\left(2 N^{2}+2 N-1\right)$. Likewise, in the limit $q \rightarrow \infty$,

$$
f(N, q \rightarrow \infty) \sim \frac{1}{q^{8}}\left[\zeta(3)+\frac{1}{N^{3}}+\frac{\psi_{2}(N)}{2}\right] .
$$

Given Eq. (4.3), we can readily write a sum rule for $f(N, q)$ since the total kinetic-energy $T$, from the virial theorem $T=-E$ for a Coulomb field, is

$$
T=Z^{2} N .
$$

But in terms of $n(N, Z, p)$

$$
T=\int_{0}^{\infty} n(N, Z, p) \frac{p^{2}}{2} 4 \pi p^{2} d p=\frac{32}{\pi} Z^{-3} \int_{0}^{\infty} Z^{5} f(N, q) q^{4} d q,
$$

showing from comparison of Eqs. (4.6) and (4.7) that

$$
\int_{0}^{\infty} f(N, q) q^{4} d q=\frac{\pi}{32} N
$$

Other finite moments of $f$ can also be calculated from known results [see, for example, Ref. [22] and other references given there] and are listed in Table I.

It hardly needs emphasis that as $N \rightarrow \infty$, the result (4.7) shows that this moment of $f(N, q)$ diverges, which in fact is due to the singular behavior of $f_{\infty}(q)$ at $q=0$. However, some higher moments remain finite in this limit.

To conclude, let us mention in this context that the Fourier transform of $n_{N}(p)$, namely, $\widetilde{n}_{N}(r)$, can be evaluated in the present case. For $N$ closed shells, we find

$$
\widetilde{n}(r)=2\left[1-r \frac{\partial}{\partial r}+\frac{r^{2}}{3} \frac{\partial^{2}}{\partial r^{2}}\right] \sum_{m=1}^{N} m^{2}[\exp (-Z r)]^{1 / m} .
$$

We illustrate in Fig. $1 \widetilde{n}(r)$ for the case $Z=92, N=10$ previously considered in some detail in Ref. [20]. Evaluation of $\widetilde{t}(r)$ from Eq. (2.7) gives

$$
\tilde{t}(r)=\left[1-r \frac{\partial}{\partial r}-\frac{r^{2}}{3} \frac{\partial^{2}}{\partial r^{2}}\right] \sum_{m=1}^{N} Z^{2}[\exp (-Z r)]^{1 / m} .
$$




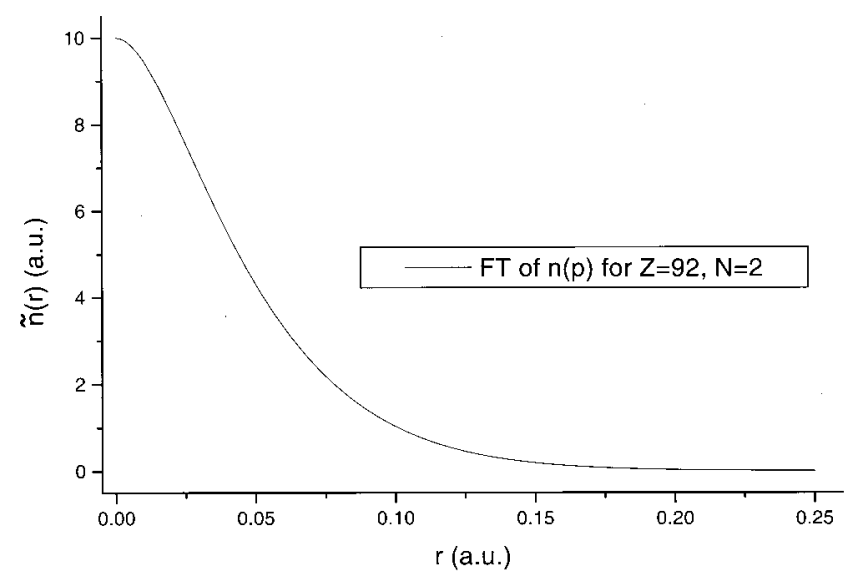

FIG. 1. The Fourier transform $\widetilde{n}(r)$ of $n(p)$ for the case $Z$ $=92$, with ten electrons in a bare Coulomb field (see also Ref. [20]).

Making use of Eq. (4.8), we can easily verify that $\widetilde{t}(r)$ satisfies Eq. (2.9).

\section{PERTURBATIVE TREATMENT OF A LOCALIZED POTENTIAL $V(r)$ IN AN INITIALLY UNIFORM ELECTRON GAS}

In their early work, March and Murray [23] were concerned with calculating the "displaced" electron density $\Delta \rho(\mathbf{r})$ defined by

$$
\Delta \rho(\mathbf{r})=\rho(\mathbf{r})-\rho_{0},
$$

where $\rho_{0}$ is the density of the initially uniform electron gas while $\rho(\mathbf{r})$ is the density after introducing the "perturbing" potential $V(\mathbf{r})$, which is assumed to be localized such that $\int V(\mathbf{r}) d \mathbf{r}$ through the whole of space exists. Their result to first order in $V(\mathbf{r})$ was

$$
\Delta \rho_{1}(\mathbf{r})=-\frac{k_{F}^{2}}{2 \pi^{2}} \int \frac{V\left(\mathbf{r}^{\prime}\right) j_{1}\left(2 k_{F}\left|\mathbf{r}-\mathbf{r}^{\prime}\right|\right)}{\left|\mathbf{r}-\mathbf{r}^{\prime}\right|^{2}} d \mathbf{r}^{\prime},
$$

where $k_{F}$ is the Fermi wave number of the electron gas, related to the uniform density $\rho_{0}$ by

$$
\rho_{0}=\frac{k_{F}^{3}}{3 \pi^{2}}
$$

The perturbed first-order density matrix $\Delta \gamma\left(\mathbf{r}, \mathbf{r}^{\prime}\right)$ was also obtained by March and Murray [23] in a perturbation series to all orders in $V(\mathbf{r})$, namely,

$$
\Delta \gamma\left(\mathbf{r}, \mathbf{r}^{\prime}\right)=\sum_{j=1}^{\infty} \Delta \gamma_{j}\left(\mathbf{r}, \mathbf{r}^{\prime}\right)
$$

Below we consider their explicit first-order form,

$$
\Delta \gamma_{1}\left(\mathbf{r}, \mathbf{r}^{\prime}\right)=-\frac{k_{F}^{2}}{2 \pi^{2}} \int \frac{V\left(\mathbf{r}^{\prime \prime}\right) j_{1}\left(k_{F}\left\{\left|\mathbf{r}-\mathbf{r}^{\prime \prime}\right|+\left|\mathbf{r}^{\prime \prime}-\mathbf{r}^{\prime}\right|\right\}\right)}{\left|\mathbf{r}-\mathbf{r}^{\prime \prime}\right|\left|\mathbf{r}^{\prime \prime}-\mathbf{r}^{\prime}\right|} d \mathbf{r}^{\prime \prime}
$$

or

$$
\begin{aligned}
& \Delta \gamma_{1}\left(\mathbf{r}^{\prime}-\mathbf{r}, \mathbf{r}^{\prime}\right) \\
& \quad=-\frac{k_{F}^{2}}{2 \pi^{2}} \int \frac{V\left(\mathbf{r}^{\prime \prime}\right) j_{1}\left(k_{F}\left\{\left|\mathbf{r}^{\prime}-\mathbf{r}^{\prime \prime}-\mathbf{r}\right|+\left|\mathbf{r}^{\prime \prime}-\mathbf{r}^{\prime}\right|\right\}\right)}{\left|\mathbf{r}^{\prime}-\mathbf{r}^{\prime \prime}-\mathbf{r}\right|\left|\mathbf{r}^{\prime \prime}-\mathbf{r}^{\prime}\right|} d \mathbf{r}^{\prime \prime} .
\end{aligned}
$$

Then it follows that

$$
\begin{aligned}
\Delta \widetilde{n}(r)= & \int \Delta \gamma_{1}\left(\mathbf{r}^{\prime}-\mathbf{r}, \mathbf{r}^{\prime}\right) d \mathbf{r}^{\prime} \\
= & -\frac{k_{F}^{2}}{2 \pi^{2}} \int d \mathbf{r}^{\prime \prime} V\left(\mathbf{r}^{\prime \prime}\right) \int d \mathbf{r}^{\prime} \\
& \times \frac{j_{1}\left(k_{F}\left\{\left|\mathbf{r}^{\prime}-\mathbf{r}^{\prime \prime}-\mathbf{r}\right|+\left|\mathbf{r}^{\prime \prime}-\mathbf{r}^{\prime}\right|\right\}\right)}{\left|\mathbf{r}^{\prime}-\mathbf{r}^{\prime \prime}-\mathbf{r}\right|\left|\mathbf{r}^{\prime \prime}-\mathbf{r}^{\prime}\right|},
\end{aligned}
$$

or

$$
\begin{aligned}
\Delta \widetilde{n}(r) & =-\frac{k_{F}^{2}}{2 \pi^{2}} \int d \mathbf{r}^{\prime \prime} V\left(\mathbf{r}^{\prime \prime}\right) \int d \mathbf{R} \frac{j_{1}\left(k_{F}\{|\mathbf{R}-\mathbf{r}|+R\}\right)}{|\mathbf{R}-\mathbf{r}| R} \\
& =-\frac{k_{F}^{2}}{2 \pi^{2}} f(\mathbf{r}) \int d \mathbf{r}^{\prime \prime} V\left(\mathbf{r}^{\prime \prime}\right)
\end{aligned}
$$

What seems remarkable here is that the r-space form of $\Delta \widetilde{n}(r)$ is independent of the detailed shape of the localized potential $V(\mathbf{r})$, which simply fixes the magnitude of $\Delta \tilde{n}(r)$. It would, of course, be important for the future if such simplification could be proved to occur also in higher terms of the March-Murray perturbation theory.

\section{SUMMARY AND FUTURE DIRECTIONS}

Conventional approaches to kinetic energy are (i) densityfunctional theory and (ii) via momentum space, or equivalently via the first-order density matrix $\gamma\left(\mathbf{r}, \mathbf{r}^{\prime}\right)$ using the Fourier transform relation (2.10) with $n(\mathbf{p})$. Here, we have introduced a third approach via the quantities $\widetilde{n}(\mathbf{r})$ and $\widetilde{t}(\mathbf{r})$ defined in Eqs. (2.4) and (2.7), respectively, and related via a "Poisson-like" Eq. (2.9). This equation strongly suggests that $\tilde{t}(\mathbf{r})$ will be simpler than $\tilde{n}(\mathbf{r})$ just as in Poisson's equation, the charge density is (usually) simpler than the electrostatic potential it generates.

As examples of the present approach, we have discussed in turn isotropic harmonic confinement and Fermions moving in a bare Coulomb field, both these examples being treated for an arbitrary number of closed shells. Following these two cases dealing with bound states, the third area taken is that of a localized potential $V(\mathbf{r})$ introduced into an initially uniform electron gas, using the perturbative theory of March and Murray calculated to all orders in $V$. We show explicitly to first order that the present route using $\widetilde{n}(\mathbf{r})$ and $\tilde{t}(\mathbf{r})$ is remarkable in its analytical simplicity.

Of course, it is very important for the future that one transcends the examples considered of independent Fermions. Therefore, in Appendix A, we have considered the two- 
electron Hookean atom, for which the first-order density matrix $\gamma\left(\mathbf{r}, \mathbf{r}^{\prime}\right)$ is known in integral form in the presence of electron-electron interactions. This allows $\widetilde{n}(\mathbf{r})$ to be also expressed in integral form but, so far, we have not succeeded in evaluating the integrals. However, there should be no difficulty in evaluating $\widetilde{n}(\mathbf{r})$, should the need arise in the future, by numerical multidimensional integration. Furthermore, one can form $\boldsymbol{\nabla}_{r}^{2} \widetilde{n}(\mathbf{r})$ by interchanging the order of the operation $\nabla_{\mathbf{r}}^{2}$ and the multidimensional integration involved to find $\widetilde{n}(\mathbf{r})$. Hence, using the Poisson-like form (2.9) relating $\widetilde{t}(\mathbf{r})$ and $\nabla_{\mathbf{r}}^{2} \widetilde{n}(\mathbf{r})$, the calculation of $\tilde{t}(\mathbf{r})$ should also be feasible, if needed in the future. Also, it would be of interest, we believe, to extend the results of the Appendix to the He-like series of atomic ions with nuclear charge $\mathrm{Ze}$, but then one must have recourse to correlated wave functions since $\gamma\left(\mathbf{r}, \mathbf{r}^{\prime}\right)$ is no longer known, in contrast to the two-electron Hookean atom, in closed form.

\section{ACKNOWLEDGMENTS}

I.A.H. wishes to acknowledge support from the Flemish Science Foundation (FWO) under Grant No. G.0347.97. We also thank the University of Antwerp (RUCA) for its support in the framework of the Visiting Professors Program. This work is also supported by the Concerted Action Program of the University of Antwerp.

\section{APPENDIX A: HOOKEAN ATOM WITH FORCE CONSTANT $k=1 / 4$}

The single-particle density matrix $\gamma\left(\mathbf{r}, \mathbf{r}^{\prime}\right)$ for the Hookean atom with force constant $k=1 / 4$ has been used by March, Amovilli, and Klein [24] to show that the electron density $\rho(r)$ is determined by the pair function $n_{2}\left(\mathbf{r}, \mathbf{r}^{\prime}\right)$ at coincidence, i.e., $\mathbf{r}^{\prime}=\mathbf{r}$. Here, we utilize $\gamma\left(\mathbf{r}, \mathbf{r}^{\prime}\right)$ to find the quantity $\widetilde{n}(\mathbf{r})$ defined in Sec. II, namely, the Fourier transform of the momentum density. [25])

The explicit form of $\gamma\left(\mathbf{r}, \mathbf{r}^{\prime}\right)$ is (see, for example, Ref.

$$
\begin{aligned}
\gamma\left(\mathbf{r}, \mathbf{r}^{\prime}\right)= & 2 C^{2} \exp \left(-\frac{r^{2}+r^{\prime 2}}{4}\right) \int\left(1+\frac{\left|\mathbf{r}-\mathbf{r}^{\prime \prime}\right|}{2}\right) \\
& \times\left(1+\frac{\left|\mathbf{r}^{\prime}-\mathbf{r}^{\prime \prime}\right|}{2}\right) \exp \left(-\frac{r^{\prime \prime 2}}{2}\right) d \mathbf{r}^{\prime \prime},
\end{aligned}
$$

where $C=\left[2 \pi^{5 / 4}(5 \sqrt{\pi}+8)^{1 / 2}\right]^{-1}=.0291122$ a.u. Since from Eq. (2.11), $\widetilde{n}(\mathbf{r})$ is a contracted form of $\gamma\left(\mathbf{r}^{\prime}-\mathbf{r}, \mathbf{r}^{\prime}\right)$ we note first that this latter quantity is explicitly

$$
\begin{aligned}
\gamma\left(\mathbf{r}^{\prime}-\mathbf{r}, \mathbf{r}^{\prime}\right)= & 2 C^{2} \exp \left(-\frac{\left|\mathbf{r}^{\prime}-\mathbf{r}\right|^{2}+r^{\prime 2}}{4}\right) \\
& \times \int\left(1+\frac{\left|\mathbf{r}^{\prime}-\mathbf{r}-\mathbf{r}^{\prime \prime}\right|}{2}\right)\left(1+\frac{\left|\mathbf{r}^{\prime}-\mathbf{r}^{\prime \prime}\right|}{2}\right) \\
& \times \exp \left(-\frac{r^{\prime \prime 2}}{2}\right) d \mathbf{r}^{\prime \prime}
\end{aligned}
$$

If we put $\mathbf{R}=\mathbf{r}^{\prime \prime}-\mathbf{r}^{\prime}$ in Eq. (A2) we find

$$
\begin{aligned}
\gamma\left(\mathbf{r}^{\prime}-\mathbf{r}, \mathbf{r}^{\prime}\right)= & 2 C^{2} \exp \left(-\frac{\left|\mathbf{r}^{\prime}-\mathbf{r}\right|^{2}+r^{\prime 2}}{4}\right) \int\left(1+\frac{|\mathbf{r}+\mathbf{R}|}{2}\right) \\
& \times\left(1+\frac{\mathbf{R}}{2}\right) \exp \left(-\frac{\left(\mathbf{r}^{\prime}+\mathbf{R}\right)^{2}}{2}\right) d \mathbf{R} .
\end{aligned}
$$

We assume we can interchange the order of integration to write

$$
\begin{aligned}
\widetilde{n}(\mathbf{r})= & \int \gamma \mathbf{r}^{\prime}-\mathbf{r}, \mathbf{r}^{\prime} d \mathbf{r}^{\prime} \\
= & 2 C^{2} \int d \mathbf{R}\left(1+\frac{|\mathbf{r}+\mathbf{R}|}{2}\right)\left(1+\frac{\mathbf{R}}{2}\right) \int d \mathbf{r}^{\prime} \\
& \times \exp \left(-\frac{\left|\mathbf{r}^{\prime}-\mathbf{r}\right|^{2}+r^{\prime 2}}{4}\right) \exp \left(-\frac{\left(\mathbf{r}^{\prime}+\mathbf{R}\right)^{2}}{2}\right) .
\end{aligned}
$$

Putting $\mathbf{S}=\mathbf{R}+\mathbf{r}^{\prime}$, we can write the second integral appearing in Eq. (A4), denoted by $I(\mathbf{r}, \mathbf{R})$ below, as

$$
I(\mathbf{r}, \mathbf{R})=\int d \mathbf{S} \exp \left(-\frac{\left\{\mathbf{S}-\mathbf{R}-\left.\mathbf{r}\right|^{2}+|\mathbf{S}-\mathbf{R}|^{2}\right\}}{4}\right) \exp \left(-S^{2} / 2\right),
$$

so that

$$
\widetilde{n}(\mathbf{r})=2 C^{2} \int d \mathbf{R}\left(1+\frac{|\mathbf{r}+\mathbf{R}|}{2}\right)\left(1+\frac{\mathbf{R}}{2}\right) I(\mathbf{r}, \mathbf{R}) .
$$

This quite explicit expression for $\widetilde{n}(\mathbf{r})$, by numerical integration, could be compared with the harmonic confinement result (3.3) for $M=0$ should it prove useful in the future, but naturally with the same Hookean force constant.

\section{APPENDIX B: RELATION OF $J(q)$ to $\widetilde{n}(r)$}

Making use of Eq. (2.2) in Eq. (2.3), we have

$$
J(q)=2 \pi \int_{q}^{\infty} p n(p) d p .
$$

Since we can write $n(p)$ in terms of $\widetilde{n}(r)$ as

$$
n(p)=\int \widetilde{n}(r) \exp (i \mathbf{p} \cdot \mathbf{r}) d \mathbf{r},
$$

$J(q)$ is just 


$$
\begin{aligned}
J(q) & =8 \pi^{2} \int_{q}^{\infty} p d p \int_{0}^{\infty} \tilde{n}(r) \frac{\sin (p r)}{p r} r^{2} d r \\
& =8 \pi^{2} \int_{0}^{\infty} r \widetilde{n}(r)\left[\int_{q}^{\infty} \sin (p r) d p\right] d r .
\end{aligned}
$$

If we let

$$
F(q, r, \lambda)=\int_{p}^{\infty} \sin (p r) \exp (-\lambda p r) d p
$$

then $\lim _{\lambda \rightarrow 0} F(q, r, \lambda)=\cos (q r) / r$, so that

$$
J(q)=8 \pi^{2} \int_{0}^{\infty} \tilde{n}(r) \cos (q r) d r .
$$

As for $n(p)$, this equation shows that $\widetilde{n}(r)$ is directly accessible from an experimentally measured Compton profile.
[1] L. H. Thomas, Proc. Cambridge Philos. Soc. 23, 542 (1926).

[2] E. Fermi, Z. Phys. 48, 73 (1928).

[3] N. H. March, Adv. Phys. 6, 1 (1957).

[4] A. Konya, Acta Phys. Hung. 1, 12 (1949).

[5] C. A. Coulson and N. H. March, Proc. Phys. Soc. London, Ser. A 63, 367 (1950).

[6] N. H. March, J. Chem. Phys. 79, 3404 (1983).

[7] V. Fock, Z. Phys. 98, 145 (1935).

[8] R. McWeeny, Methods of Molecular Quantum Mechanics, 2nd ed. (Academic, London, 1989).

[9] V. H. Smith, in Electron, Spin and Momentum Densities and Chemical Reactivity, edited by P. G. Mezey and B. Robertson (Kluwer, Dordrecht, The Netherlands, 1998).

[10] K. Husimi, Proc. Phys. Math. Soc. Jpn. 22, 264 (1940).

[11] G. P. Lawes and N. H. March, J. Chem. Phys. 71, 1007 (1979).

[12] J. Bardeen, Phys. Rev. 49, 653 (1936).

[13] J. S. Brown, R. C. Brown, and N. H. March, Phys. Lett. 46A, 403 (1974).
[14] N. H. March, Phys. Rev. B 61, 5011 (2000).

[15] N. R. Kestner and O. Sinanoglu, Phys. Rev. 128, 2687 (1962).

[16] W. E. Duncanson and C. A. Coulson, Proc. Phys. Soc., London, Sect. A 57, 190 (1945).

[17] I. A. Howard and N. H. March, J. Phys. A (to be published).

[18] P. M. Morse and H. Feshbach, Methods of Theoretical Physics, Vol. II (MacGraw-Hill, New York, 1953), Chap. 12.

[19] Handbook of Mathematical Functions, edited by M. Abramowitz and I. A. Stegun (Dover, New York, 1970).

[20] I. A. Howard, N. H. March, and V. E. Van Doren, Phys. Rev. A 63, 062501 (2001).

[21] O. J. Heilmann and E. H. Lieb, Phys. Rev. A 52, 3628 (1995).

[22] N. H. March, J. Chem. Phys. 79, 3404 (1983).

[23] N. H. March and A. M. Murray, Proc. R. Soc. London, Ser. A 261, 119 (1961).

[24] N. H. March, C. Amovilli, and D. J. Klein, Chem. Phys. Lett. 325, 645 (2000).

[25] Z. Qian and V. Sahni, Phys. Rev. A 57, 2527 (1998). 